\title{
ACONTECIMIENTOS VITALES Y SINTOMATOLOGÍA DEPRESIVA EN POBLACIÓN ADOLESCENTE
}

\author{
ESTRElla Ferreira, Roser Granero, ZAHARA NoOrian, Kelly Romero \\ Y EDELMIRA DOMÈNECH-LLABERIA
}

Facultad de Psicología, Universidad Autónoma de Barcelona, Barcelona, España

\begin{abstract}
Resumen: El presente estudio exploró la relación entre acontecimientos vitales y su nivel de impacto percibido con sintomatología depresiva en población adolescente. Se evaluaron 1.061 adolescentes de ambos sexos (13-16 años) a través de autoinformes (Life Events Checklist and Children's Depression Inventory). Los resultados mostraron que los acontecimientos vitales positivos disminuyen el nivel de sintomatología depresiva, mientras que los negativos la aumentan. El nivel de impacto percibido por los acontecimientos negativos aumenta el grado de sintomatología depresiva, y se observa interacción significativa con la procedencia del sujeto (impacto percibido mayor en población autóctona). Considerando los subtipos de acontecimientos, los familiares, escolares y de salud negativos aumentan el nivel de sintomatología depresiva mientras que los sociales la disminuyen. En cuanto al impacto percibido en estos subtipos, los resultados dependen del tipo de acontecimiento y de la edad de los sujetos.
\end{abstract}

Palabras clave: Sucesos vitales; sintomatología depresiva; estrés percibido; adolescencia.

\section{Life events and depressive symptoms in adolescents}

\begin{abstract}
This study examinated the association between life events and the level of their perceived impact with depressive symptoms in adolescents. A total of 1,061 adolescents of both sexes (13-16 years) were evaluated through self-report questionnaires (Life Events Checklist and Children's Depression Inventory). Results showed that positive life events decrease the level of depressive symptoms, while negative ones increase them. The perceived impact level of negative life events increases the degree of depressive symptoms, while a significant interaction with students' origin was observed (native people perceived a greater impact). Considering the subtypes of life events, family, school and negative health increased the level of depressive symptoms while it decreased with social life events. Results on the perceived impact of these subtypes depend on the type of event and the age of the subjects.
\end{abstract}

Keywords: Life events; depressive symptoms; perceived stress; adolescence.

\section{INTRODUCCIÓN}

A lo largo de todo el ciclo vital el ser humano está expuesto a situaciones, estímulos o circunstancias que ocurren de forma inesperada,

Recibido: 17 julio 2011; aceptado: 27 enero 2012.

Correspondencia: Edelmira Domènech-Llabería, Universidad Autónoma de Barcelona, Facultad de Psicología, Departamento de Psicología Clínica y de la Salud, 08193 Bellaterra, Barcelona, España.

Correo-e: edelmira.domenech@uab.cat que provocan cambios o reajustes en la vida del sujeto, y que por ende pueden alterar potencialmente su estado físico y/o mental (Goodyer, 1990). Éstas se han denominado life events, sucesos vitales o acontecimientos vitales (AV). Los AV pueden categorizarse como positivos o negativos, y su percepción como tales depende de la interrelación de los factores personales del sujeto y los factores del entorno.

En la mayoría de las investigaciones sobre este tema se estudian los AV negativos, y se relacionan con el aumento de las alteraciones 
emocionales en niños y adolescentes, pues son etapas, sobretodo la adolescencia, en las que tienen lugar infinidad de cambios biológicos, escolares, sociales, familiares, etc. y por tanto puede considerarse una etapa de vulnerabilidad (Finlay-Jones y Brown, 1981; Goodyer, Kolvin y Gatzanis, 1985; Johnson, 1986; Larson y Ham, 1993). Diversos estudios con adolescentes (Williamson et al., 1998; Olsson, Nordström, Arinell y Von Knorring, 1999; Goodyer, Herbert y Tamplin, 2000) han puesto de manifiesto los efectos de los AV en el curso de la depresión, y han encontrado que los adolescentes deprimidos experimentaron significativamente más acontecimientos adversos antes del inicio de un cuadro depresivo, que los adolescentes del grupo control sin depresión.

Los AV también han sido estudiados de forma cualitativa. Algunos autores se limitan a hacer un análisis de cada uno de los ítems de los diferentes cuestionarios de evaluación, sin agruparlos por subescalas, como es el caso de Olsson et al. (1999) que relacionan cada ítem del Life Events Questionnaire (Coddington, 1972) con las diferentes manifestaciones de depresión, obteniendo que cinco ítems son significativamente más comunes entre los adolescentes depresivos, cuatro de los cuales hacen referencia a las relaciones familiares y de amistad, y el quinto al tipo de problema de salud que se padezca. Tiet et al. (2001), encuentran los mismos resultados en su análisis de los ítems del Life Events Checklist (LEC; Johnson y McCutcheon, 1980), a excepción de la enfermedad propia que en este estudio no tiene relación significativa con el trastorno de depresión mayor en niños y adolescentes.

Otros autores realizan un análisis cualitativo a partir de subtipos de AV. Mayer et al. (2009) clasifican 26 acontecimientos estresantes en cinco grupos: salud de los padres, muerte de seres cercanos, sociodemográficos, intrafamiliares y 'otros' en los que engloban abusos, problemas judiciales, y dificultades o problemas escolares. Jiménez et al. (2008) realizan una distinción más sencilla a partir del Inventario de Acontecimientos Vitales Estresantes de Oliva, Jiménez, Parra, y Sánchez-Queija (2008), separándolos entre AV del ámbito familiar y AV del grupo de iguales, incluyendo en este último los relacionados con el contexto escolar. Williamson, Birmaher, Dahl y Ryan (2005), diferencian en el LEC entre tres tipos de $\mathrm{AV}$, los dependientes, que son aquellos que dependen del comportamiento del adolescente (p.ej., 'ser expulsado del centro escolar'), los independientes, que son aquellos que no dependen del comportamiento del adolescentes (p.ej., 'tu padre ha sido despedido del trabajo'), y los de pérdida, que pueden ser tanto dependientes como independientes, pero hacen referencia a una pérdida ya sea material (p.ej., 'cambio de domicilio') como emocional (p.ej., 'muerte de un familiar'). Puskar, TusaieMumford, Sereika, y Lamb (1999), realizan la clasificación a partir del LEC en AV familiares, $\mathrm{AV}$ con los iguales (escolares y sociales) y otros (relacionados con la justicia), encontrando que son las discusiones con los padres y los problemas con los compañeros los AV más relacionados con la sintomatología depresiva.

De entre las diferentes clasificaciones, en este estudio utilizaremos la determinada por Moreno, Del Barrio y Mestre (1996), en la que diferencian entre AV familiares, escolares, de salud, y sociales. Estas autoras realizaron una adaptación del Inventario de Acontecimientos Vitales en Infancia y Adolescencia y clasificaron los 81 ítems en 4 subescalas para relacionar los acontecimientos vitales estresantes con niveles de ansiedad en población española.

No se pueden considerar los AV sin tener en cuenta la resistencia a su impacto o 'resiliencia', entendida como el fenómeno caracterizado por el desarrollo de modelos de adaptación positiva o competencia en contextos de riesgo o adversidad (Egeland, Carlson y Sroufe, 1993; Lázaro, 2009; Masten y Reed, 2002; Rutter, 2007). Existen varias investigaciones que hacen referencia a ello. Smith (2009) encontró que las personas con mayor resiliencia ante los acontecimientos presentaban menores trastornos emocionales que aquellos cuya resiliencia era baja. Hjemdal, Aune, Reinfjell, Stiles, y Friborg (2007) encontraron, así mismo, que la resiliencia es un factor protector de los trastornos depresivos, pues las personas con alta resiliencia presentaban menor sintomatología depresiva ante los AV.

En el estudio de los AV y su relación con las alteraciones emocionales, un aspecto importante es la comorbilidad común con los trastornos 
de ansiedad. Son diversos los autores que han documentado dicha relación (Kovacs, 1990; Weller y Weller, 2000; Sandín, Valiente y Chorot, 2009) y, entre otros, Avenevoli, Stolar, Li, Dierker y Merikangas (2001) encontraron que aproximadamente del $20 \%$ al $75 \%$ de los adolescentes con trastorno depresivo tenían también un trastorno de ansiedad comórbido.

Los objetivos de esta investigación son: 1) conocer si la vivencia de AV negativos aumenta la presencia de sintomatología depresiva, y si por el contrario, la vivencia de AV positivos la disminuye; 2) analizar si dicha sintomatología y nivel de afectación varían en función del nivel de impacto percibido de los $\mathrm{AV}$, tanto positivos como negativos; 3 ) explorar qué subtipo/s de AV (familiar, social, escolar o de salud) está/n más estrechamente relacionado/s con la presencia y nivel de afectación de sintomatología depresiva; y 4) valorar si el grado de sintomatología depresiva se relaciona con el nivel de afectación o impacto percibido por los AV.

\section{MÉTODO}

\section{Participantes}

Este estudio se realizó en la población de Rubí, en la provincia de Barcelona. Dicha población tiene censados a 72.987 habitantes, de los cuales, según el último informe del Instituto Nacional de Estadística (INE 2009), 11.593 (16\%) son inmigrantes, siendo 2.071 de éstos menores de 16 años. Este estudio de campo iba dirigido concretamente a todos los alumnos escolarizados durante el curso 2009/2010 en 2 . $^{\circ}$ y $3 .^{\circ}$ de Educación Secundaria Obligatoria (ESO). La población inicial estaba formada por 1.324 niños escolarizados tanto en escuelas públicas como concertadas. De ellos, 43 no fueron autorizados por sus padres a participar en la investigación, 2 alumnos no quisieron colaborar, a pesar de tener la autorización de los padres, y 218 no estuvieron en los centros escolares cuando se realizaron las pruebas. La muestra final del estudio estuvo formada por 1061 sujetos (el porcentaje de participación fue del 80,1\%). El 52,7\% pertenecía al sexo masculino y el rango de edad estuvo comprendido entre los 13 y los 16 años $(M=13,92 ; D T=$ $0,78)$. Respecto a la procedencia, el $21,2 \%$ eran inmigrantes nacidos fuera de España. El 50,2\% de los participantes cursaba $2 .^{\circ}$ de la ESO, y el $49,8 \%$ a $3 .^{\circ}$. Respecto al tipo de escuela, el $63,3 \%$ procedía de colegios públicos y el $36,7 \%$ de colegios concertados. De acuerdo al índice sociodemográfico de Hollingshead (1975), que se construye a partir del nivel de estudios y la profesión de los principales cuidadores de los adolescentes, el 23,5\% de los participantes tienen un nivel socioeconómico entre medio-alto $\mathrm{y}$ alto, un $30,8 \%$ un nivel medio, y el restante $45,6 \%$ niveles medio-bajo o bajo. La Tabla 1 presenta la descripción completa de la muestra.

Tabla 1. Características sociodemográficas de la muestra

\begin{tabular}{llc}
\hline Edad (años), $n$ (\%) & 13 años & $331(31,2 \%)$ \\
& 14 años & $510(48,1 \%)$ \\
& 15 años & $192(18,1 \%)$ \\
& 16 años & $27(2,5 \%)$ \\
Edad (años); Media (DT) & $13,92(0,76)$ & \\
Sexo, $n$ (\%) & Masculino & $559(52,7 \%)$ \\
Curso escolar, $n(\%)$ & Femenino & $502(47,3 \%)$ \\
& $2 .{ }^{\circ}$ ESO & $533(50,2 \%)$ \\
Tipo de escuela, $n(\%)$ & $3 .^{\circ}$ ESO & $528(49,8 \%)$ \\
& Público & $672(63,3 \%)$ \\
Nivel socioeconómico, $n(\%)$ & Concertado & $389(36,7 \%)$ \\
(Hollingshead, 1975) & Medio-Alto & $232(22,5 \%)$ \\
& Medio & $317(30,8 \%)$ \\
& Medio-Bajo & $397(38,6 \%)$ \\
& Bajo & $73(7,1 \%)$ \\
& España & $834(78,8 \%)$ \\
Lugar de procedencia, $n(\%)$ & América latina & $159(15,0 \%)$ \\
& África & $47(4,4 \%)$ \\
& Asia & $14(1,3 \%)$ \\
& Europa & $5(0,5 \%)$ \\
\hline
\end{tabular}

Nota. ESO-Enseñanza Secundaria Obligatoria.

\section{Instrumentos de evaluación}

Life Events Checklist (LEC; Johnson y McCutcheon, 1980). Se utilizó para la medición de los acontecimientos vitales. El LEC está compuesto por 46 ítems que representan diferentes 
acontecimientos vitales (p.ej., 'Separación de tus padres' y 'Muerte de un familiar'). A los participantes se les pregunta por los acontecimientos vividos en los últimos 12 meses, y los sujetos han de responder en qué medida los han vivido como positivos o negativos, y qué impacto les han causado (en una escala ordinal con 4 alternativas que van de $0=$ ningún impacto a $3=$ gran impacto). El estudio de la fiabilidad test-retest en un intervalo temporal de 2 semanas concluyó una adecuada fiabilidad, con correlaciones del test-retest para el total de acontecimientos positivos y negativos de 0,69 y 0,72 respectivamente (Brand y Johnson, 1982). Esta escala, permitió obtener en este estudio las siguientes puntuaciones: a) número total de acontecimientos vitales que ha vivido el sujeto; b) número total de acontecimientos positivos y negativos; c) puntuación total del impacto percibido como positivo (se obtuvo como la suma no ponderada del impacto de los acontecimientos autopercibidos como 'positivos'); d) puntación del impacto percibido como negativo (obtenido también a partir de la suma del impacto de los acontecimientos autopercibidos como 'negativos'). Se realizó una traducción y adaptación de dicho cuestionario, y posteriormente se valoró la fiabilidad de consistencia interna obteniéndose un coeficiente alfa de Cronbach aceptable $(0,75)$. En cuanto a la traducción, en un primer momento se tradujo el cuestionario original al español y posteriormente se tradujo de nuevo al inglés (retrotraducción). Este proceso se llevo a cabo dos veces, por dos traductoras diferentes para de este modo controlar aún más la pérdida de información y así obtener la traducción final. En cuanto a la adaptación, concretamente se unificaron los ítems: a) 'Divorcio de tus padres' y 'Separación de tus padres', en 'Divorcio o separación de tus padres', pues en definitiva representan para el sujeto un evento similar; b) 'Muerte de un amigo cercano' y 'Accidente o enfermedad grave de un amigo íntimo' en 'Muerte, accidente o enfermedad grave de tu pareja o de un amigo íntimo'; c) 'Embarazo propio' y 'Embarazo de tu pareja', en 'Embarazo propio o de tu pareja'; d) 'Aborto propio' y 'Aborto de tu pareja' en 'Aborto propio o de tu pareja', pues en ambos casos (embarazo y aborto) lo que nos interesa observar es la presencia o ausencia del acontecimiento y su nivel de afectación, y no que se haya vivido en primera o en tercera persona; e) 'Formar parte de un nuevo grupo', 'Dejar de hacer deporte de equipo' y 'Hacer deporte de equipo' en 'Formar parte de un nuevo grupo (amigos, deporte, scouts)' pues en nuestra cultura el formar parte de un grupo de deportes no está tan extendido en los institutos como lo está en las sociedades anglosajonas y consideramos englobar los diferentes grupos sociales posibles a esa edad en un solo ítem; $f$ ) por la misma razón se unificaron los ítems ' $\mathrm{Ob}$ tener un premio especial' y 'Especial reconocimiento en deporte' en 'Obtener un premio en un concurso, campeonato, etc.'. Además, se modificó el ítem 'Cambio en el estatus económico de tus padres' convirtiéndose en 'Dificultades económicas en casa', pues los adolescentes a los que se les pasó la prueba les era más fácil de comprender el segundo enunciado. También se eliminaron del cuestionario los ítems 'Perder el trabajo' y 'Conseguir un trabajo' pues a la edad a la que se pasa este cuestionario es ilegal que los sujetos estén empleados. Finalmente, se añadieron los siguientes cinco ítems: 1) 'Haber sufrido aislamiento, rechazo o ridiculizaciones frecuentes por parte de tus compañeros'; 2) 'Haber tenido problemas con el alcohol, las drogas o con otro tipo de adicción'; 3) 'Un familiar cercano ha creado problemas por algún tipo de adicción (alcohol, drogas, juego u otros)'; 4) 'Haber sido víctima de algún delito (robo, agresión, etc.)'; y, 5) 'Haber sido víctima de abuso sexual en algún momento de toda tu vida'. De este modo y tras los cambios señalados, esta versión del LEC queda compuesta por un total de 40 ítems. Y para finalizar la adaptación, previo al inicio del estudio, el cuestionario se aplicó en un centro escolar próximo al municipio y con las mismas características que los centros donde se realizó el estudio. De este instrumento se obtiene información cualitativa, por lo que cuando se obtienen porcentajes se hace referencia a la presencia de dichos acontecimientos ya se hayan presentado éstos una única vez o de forma repetida.

Children's Depression Inventory (CDI; Kovacs, 1983). Es un cuestionario de autoinforme que evalúa la sintomatología depresiva en niños y adolescentes entre 7 y 17 años. Consta de un total de 27 ítems en los que el sujeto ha de es- 
coger entre tres alternativas (en una escala ordinal con puntuación de 0 a 2), la que mejor describa cómo se ha sentido en las últimas dos semanas (p.ej., 'Estoy triste de vez en cuando', 'Estoy triste muchas veces', o 'Estoy triste siempre'). La puntuación global del CDI se obtiene a partir de una suma directa de las respuestas dadas a cada ítem, y está comprendida dentro del rango 0 a 54 puntos. El punto de corte que determina si la puntuación ha de considerarse «de riesgo» se sitúa en 17 en población española, con una sensibilidad de 0,84 y una especificidad de 0,72 (Domènech, Subirà y Cuxart, 1996). El CDI ha mostrado buena consistencia interna tanto en muestras clínicas como en noclínicas, con valores alfa de Cronbach por encima del 0,7 (Kovacs, 1992). Concretamente, en población española el alfa fue de 0,8 (Del Barrio, Moreno-Rosset y López-Martínez, 1999; Mestre, Frías y García-Ros, 1992). En el presente estudio se ha obtenido un valor de 0,83 .

Screen for Child Anxiety Related Emotional Disorders (SCARED; Birmaher, Khetarpal y Cully, 1997). Es un cuestionario de autoinforme que mide los síntomas de ansiedad en niños y adolescentes de entre 8 y 18 años. Está compuesto por 41 ítems y 5 subescalas: ansiedad generalizada (p.ej., 'Me preocupo por todo'), ansiedad de separación (p.ej., 'No me gusta estar lejos de mi familia'), trastorno por pánico (p.ej., 'Cuando me asusto mi corazón va más deprisa'), fobia social (p.ej., 'No me gusta estar con personas que no conozco demasiado') y fobia escolar (p.ej., 'Me asusta ir a la escuela'). Niños y adolescentes han de responder con qué frecuencia experimentan dichos síntomas mediante una escala ordinal con 3 alternativas de respuesta $(0=$ casi nunca, $1=$ a veces, y $2=$ casi siempre). La puntuación total se obtiene a través de la suma directa, y está comprendida dentro del rango 0 a 82. El SCARED ha mostrado excelente consistencia interna, con un alfa de Cronbach igual a 0,95 en población extranjera (Birmaher et al., 1997; Muris et al., 1998). En población española, la consistencia interna fue también (alfa $=0,83)$, así como la fiabilidad test-retest con puntuaciones entre $r=0,55 \mathrm{y} r$ $=0,72$ (Doval y Domènech, 2011). Para este estudio se ha obtenido también un valor alfa elevado $(=0,85)$. Es necesario señalar que este instrumento fue utilizado en el presente estudio únicamente para controlar la sintomatología ansiosa, pues en los análisis estadísticos será tratada como una posible covariable.

Variables sociodemográficas. Para completar la información sociodemográfica se elaboró ad-hoc un cuestionario, en el que se recogió información concerniente a la edad, sexo, lugar de procedencia, curso escolar y tipo de centro escolar (público-concertado). Además, se incluyeron los indicadores necesarios para valorar el nivel socioeconómico de acuerdo a la escala de Hollinghead (Hollingshead, 1975), que se basa en los estudios y el trabajo actual de los padres o principales cuidadores.

\section{Procedimiento}

Tras la aprobación de la Comisión de Ética en Experimentación Humana de la Universidad Autónoma de Barcelona, con el informe favorable de la Comisión de Investigación de esta misma universidad, y con la conformidad de los Servicios Territoriales de Educación del Vallés de Cataluña, se solicitó la colaboración a la dirección de los colegios de educación secundaria de la localidad de Rubí. Una vez los centros confirmaron su colaboración se requirió el consentimiento firmado de los padres de los participantes y, finalmente el consentimiento informado verbal de los propios participantes (previamente se les explicaron los objetivos, se les informó de que la participación era voluntaria y se garantizó la confidencialidad). Los adolescentes completaron las pruebas de evaluación en el propio centro educativo, en horario escolar, y en presencia de su profesor y de dos investigadores que estuvieron presentes en el aula para resolver las posibles dudas que pudieran surgir. La evaluación duró aproximadamente entre 45 y 60 minutos.

\section{Análisis estadístico}

El análisis estadístico se realizó con el programa PASW17W (sistema SPSS). En un primer momento se realizaron correlaciones entre las variables del estudio para valorar las rela- 
ciones significativas, y a continuación se llevaron a cabo modelos de regresión lineal múltiple que permitieron valorar la relación entre los predictores «número de acontecimientos vitales estresantes» $\mathrm{y}$ «nivel de afectación/impacto por los AV vividos», con la variable dependiente «puntuación total en sintomatología depresiva (CDI total)». El ajuste de estos modelos se efectuó con el método enter, para obtener la contribución específica de cada variable independiente controlando por los efectos de los restantes predictores del modelo.

Tras ello, se repitieron los modelos esta vez utilizando un análisis jerárquico en el que para evitar posibles sesgos por otras variables externas a la investigación, y estrechamente relacionadas con las medidas analizadas, se controlaron a modo de covariables el sexo, la edad, el lugar de procedencia (inmigración) y la puntuación total en ansiedad (SCARED total). El ajuste de éstos modelos de regresión se efectuó en tres pasos: a) en primer lugar se introdujeron las covariables; b) en segundo lugar se agregaron las variables independientes del modelo (número de acontecimientos vitales estresantes y nivel de afectación/impacto); c) en tercer lugar se agregaron las interacciones de primer orden (generadas como nuevas variables) entre los predictores y las covariables sexo, edad, lugar de procedencia y puntuación total en ansiedad. Si las interacciones no eran significativas ( $p>0,05)$, se excluían del modelo y se estimaban los efectos principales de las variables independientes. En caso de interacción significativa $(p \leq 0,05)$, las interacciones se mantenían y se estimaban los efectos simples.

La capacidad predictiva global de los modelos de regresión múltiple (porcentaje de variabilidad de la respuesta atribuible al modelo) se valoró con el coeficiente $R^{2}$ ajustado, una vez excluida la contribución de las covariables.

\section{RESULTADOS}

En este estudio se ha obtenido que el 20,0\% de la población estudiada presenta sintomatología depresiva (CDI $\geq 17$ ), y que el $92,5 \%$ ha vivido al menos un $\mathrm{AV}$, encontrando que el $80,5 \%$ de los participantes han vivido acontecimientos positi- vos y el $86,1 \%$ negativos. En cuanto a las subescalas, los AV familiares son los que presentan una mayor frecuencia de respuesta con un $85,4 \%$, y el que presenta menor frecuenta es el subgrupo de salud con un $12,7 \%$ (véanse en la Tabla 2 los descriptivos para las diferentes variables).

Tabla 2. Descriptivos de las variables

\begin{tabular}{|c|c|c|}
\hline & Media $(D T)$ & $\begin{array}{c}\text { Presencia } \\
\%\end{array}$ \\
\hline Sintomatología depresiva & $11,51(6,31)$ & 20,0 \\
\hline Sintomatología ansiosa & $22,91(8,91)$ & 38,3 \\
\hline AV positivos & $2,04(1,75)$ & 80,5 \\
\hline AV negativos & $3,34(3,023)$ & 86,1 \\
\hline AV total & $5,39(3,93)$ & 92,5 \\
\hline Afectación por AV positivos & $16,13(5,21)$ & \\
\hline Afectación por AV negativos & $42,66(8,07)$ & \\
\hline Afectación por AV totales & $58,79(9,76)$ & \\
\hline AV familiar positivos & $0,72(0,93)$ & 49,3 \\
\hline AV familiar negativos & $2,04(1,86)$ & 77,2 \\
\hline AV familiar total & $2,77(2,22)$ & 85,4 \\
\hline $\begin{array}{l}\text { Afectación por AV familiar } \\
\text { positivos }\end{array}$ & $5,48(2,78)$ & \\
\hline $\begin{array}{l}\text { Afectación por AV familiar } \\
\text { negativos }\end{array}$ & $22,03(4,98)$ & \\
\hline $\begin{array}{l}\text { Afectación por AV familiar } \\
\quad \text { totales }\end{array}$ & $27,51(5,41)$ & \\
\hline AV escolar positivos & $0,48(0,569)$ & 44,3 \\
\hline AV escolar negativos & $0,67(1,008)$ & 39,9 \\
\hline AV escolar total & $1,14(1,144)$ & 67,4 \\
\hline $\begin{array}{l}\text { Afectación por AV escolar } \\
\text { positivos }\end{array}$ & $2,86(1,827)$ & \\
\hline $\begin{array}{l}\text { Afectación por AV escolar } \\
\text { negativos }\end{array}$ & $7,44(3,127)$ & \\
\hline $\begin{array}{l}\text { Afectación por AV escolar } \\
\quad \text { totales }\end{array}$ & $10,30(3,666)$ & \\
\hline AV social positivos & $0,84(1,013)$ & 51,8 \\
\hline AV social negativos & $0,53(0,848)$ & 36,2 \\
\hline AV social total & $1,37(1,536)$ & 62,6 \\
\hline $\begin{array}{l}\text { Afectación por AV social } \\
\text { positivos }\end{array}$ & $7,80(3,272)$ & \\
\hline $\begin{array}{l}\text { Afectación por AV social } \\
\text { negativos }\end{array}$ & $10,07(3,252)$ & \\
\hline $\begin{array}{l}\text { Afectación por AV social } \\
\text { totales }\end{array}$ & $17,86(4,920)$ & \\
\hline AV salud positivos & $0,01(0,126)$ & 1,3 \\
\hline AV salud negativos & $0,15(0,441)$ & 11,9 \\
\hline AV salud total & $0,16(0,468)$ & 12,7 \\
\hline $\begin{array}{l}\text { Afectación por AV salud } \\
\text { positivos }\end{array}$ & $1,94(1,872)$ & \\
\hline $\begin{array}{l}\text { Afectación por AV salud } \\
\text { negativos }\end{array}$ & $6,40(2,556)$ & \\
\hline $\begin{array}{l}\text { Afectación por AV salud } \\
\text { totales }\end{array}$ & $8,34(3,559)$ & \\
\hline
\end{tabular}


Previo a la ejecución de los modelos de regresión se observaron las correlaciones entre la variable dependiente «puntuación total en sintomatología depresiva» y las variables predictoras «número de acontecimientos vitales estre- santes» y «nivel de afectación/impacto por los AV vividos», así como las correlaciones entre todas las variables. Los resultados se encuentran indicados en la Tabla 3.

Tabla 3. Correlaciones entre las variables

\begin{tabular}{|c|c|c|c|c|c|c|c|c|c|c|c|c|c|c|c|}
\hline & 1 & 2 & 3 & 4 & 5 & 6 & 7 & 8 & 9 & 10 & 11 & 12 & 13 & 14 & 15 \\
\hline 1 Sintomatología depresiva & & & & & & & & & & & & & & & \\
\hline 2 Sintomatología ansiosa & $0,56^{* *}$ & & & & & & & & & & & & & & \\
\hline $3 \mathrm{AV}$ positivos & 0,04 & $0,09 * *$ & & & & & & & & & & & & & \\
\hline $4 \mathrm{AV}$ negativos & $0,41 * *$ & $* 0,28 * *$ & $* 0,30 * *$ & & & & & & & & & & & & \\
\hline $5 \mathrm{AV}$ total & $0,34 * *$ & $* 0,26 * *$ & $* 0,68 * *$ & * $0,90 * *$ & & & & & & & & & & & \\
\hline 6 Afectación por $\mathrm{AV}$ positivos & $-0,02$ & 0,00 & 0,03 & $-0,03$ & $-0,00$ & & & & & & & & & & \\
\hline 7 Afectación por AV negativos & $0,11^{* *}$ & $* 0,13 * *$ & $*-0,06 *$ & $0,14^{* *}$ & $* 0,07 *$ & 0,03 & & & & & & & & & \\
\hline 8 Afectación por AV totales & $0,08 * *$ & $* 0,11 * *$ & $*-0,03$ & $0,09 * *$ & $* 0,05$ & $0,56^{* *}$ & $* 0,84 * *$ & & & & & & & & \\
\hline 9 AV familiar total & $0,35^{* *}$ & $* 0,28 * *$ & $* 0,49 * *$ & $* 0,82 * *$ & $* 0,85^{* *}$ & $*_{-} 0,01$ & $0,10^{* *}$ & * $0,07 *$ & & & & & & & \\
\hline 10 Afectación por $\mathrm{AV}$ familiar totales & $0,09 * *$ & $* 0,11 * *$ & $*_{-} 0,05$ & $0,07^{*}$ & 0,03 & $0,37^{* *}$ & $* 0,67 * *$ & * $0,75^{* *}$ & $* 0,08 * *$ & & & & & & \\
\hline 11 AV escolar total & $0,24 * *$ & * $0,16^{* *}$ & $* 0,43^{* *}$ & $* 0,60 * *$ & $* 0,65^{* *}$ & $* 0,01$ & 0,03 & 0,03 & $0,37 * *$ & $*-0,00$ & & & & & \\
\hline 12 Afectación por $\mathrm{AV}$ escolar totales & 0,03 & 0,04 & 0,02 & 0,04 & 0,04 & $0,17^{* *}$ & $* 0,49 * *$ & $* 0,50 * *$ & $* 0,02$ & $0,16^{* *}$ & $* 0,07 *$ & & & & \\
\hline 13 AV social total & $0,14 * *$ & $* 0,11 * *$ & $* 0,67 * *$ & $* 0,57 * *$ & $* 0,73^{* *}$ & $*-0,00$ & 0,01 & 0,00 & $0,39 * *$ & $*-0,02$ & $0,34 * *$ & 0,03 & & & \\
\hline 14 Afectación por AV social totales & 0,02 & $0,06^{*}$ & $-0,03$ & 0,05 & 0,03 & $0,53^{* *}$ & $* 0,44 * *$ & $* 0,65^{* *}$ & $* 0,03$ & $0,20 * *$ & $* 0,02$ & 0,04 & 0,02 & & \\
\hline 15 AV salud total & $0,22 * *$ & $* 0,11 * *$ & $* 0,20 * *$ & $* 0,54 * *$ & $* 0,51^{* *}$ & $*-0,02$ & $0,07^{*}$ & 0,04 & $0,35 * *$ & $* 0,02$ & $0,28 * *$ & 0,03 & $0,40 * *$ & $* 0,02$ & \\
\hline 16 Afectación por AV salud totales & 0,02 & 0,02 & $-0,02$ & 0,05 & 0,03 & $0,36^{* *}$ & $* 0,40^{* *}$ & $* 0,53 * *$ & $* 0,03$ & $0,22 * *$ & $* 0,02$ & 0,03 & 0,00 & $0,63^{* *}$ & 0,05 \\
\hline
\end{tabular}

${ }^{*} \mathrm{p}<0.05 ; * * \mathrm{p}<0,01$.

\section{AV totales y sintomatología depresiva}

En la Tabla 4 presentamos el modelo de regresión múltiple que valora la relación entre las variables predictoras «total de sucesos vitales» (diferenciando entre positivos y negativos) y «nivel de afectación-impacto»y la variable dependiente puntuación total en sintomatología depresiva (CDI). Puesto que se ha obtenido una interacción significativa entre la variable total de AV negativos y procedencia (ser o no inmigrante, $p<0,05$ ) dicho parámetro ha sido retenido en el modelo. El modelo final indica que cada
AV percibido como positivo disminuye en 0,317 puntos la sintomatología depresiva (tanto en población española como inmigrante), mientras que cada AV negativo aumenta la sintomatología depresiva 0,713 puntos en población española y 0,378 puntos en población inmigrante.

En cuanto al nivel de afectación por AV los resultados indican que el nivel de impacto autopercibido por los sujetos no modifica significativamente la puntuación total del CDI. El modelo final de la Tabla 4 ha obtenido muy buena capacidad predictiva global, con $R_{\text {ajustada }}^{2}$ $=0,412(F=28,093 ; p<0,001)$.

Tabla 4. Asociación entre los AV y la puntuación total en el CDI

\begin{tabular}{|c|c|c|c|c|c|c|}
\hline & & $B$ & $\beta$ & $t$ & $p$ & IC $95 \%(B)$ \\
\hline Afectación total por AV positivos & & $-0,028$ & $-0,023$ & $-0,946$ & 0,344 & $-0,085-0,030$ \\
\hline Afectación total por AV negativos & & 0,008 & 0,011 & 0,449 & 0,654 & $-0,027-0,042$ \\
\hline Número total de AV positivos & & $-0,317$ & $-0,088$ & $-3,485$ & $0,001^{*}$ & $-0,496--0,139$ \\
\hline \multirow[t]{2}{*}{ Número total de AV negativos } & Españoles & 0,713 & 0,342 & 11,109 & $0,001 *$ & $0,587-0,839$ \\
\hline & Inmigrantes & 0,378 & 0,181 & 4,034 & $0,001^{*}$ & $0,194-0,561$ \\
\hline
\end{tabular}

Nota. Regresión múltiple ajustada por sexo, edad, puntuación ansiosa (SCARED) y procedencia (España u otros). IC = intervalo de confianza 


\section{Subtipos de AV y sintomatología depresiva}

La Tabla 5 presenta el modelo final que valora la relación entre la sintomatología depresiva y los subtipos de AV (familiar, escolar, social y de salud). Ninguna interacción fue significativa $(p>0,05$ en todos los casos). Por tanto se obtuvo e interpretó el modelo simple sin interacciones. Los resultados obtenidos indican que de los AV familiares tan solo los negativos se relacionan significativamente con la sintomatología depresiva, aumentando cada uno de ellos en promedio la puntuación del CDI en 0,416 puntos. Lo mismo ocurre con los AV de salud negativos $(B=0,985)$. El total de AV sociales no se relaciona con la puntuación total en el CDI. En cambio, los AV escolares sí que lo hacen, disminuyendo los autopercibidos como positivos la puntuación total de depresión $(B=-1,685)$, y aumentándola los negativos $(B=1,227)$. La capacidad predictiva global del modelo fue muy buena $R_{\text {ajustada }}^{2}=0,436(F=24,433 ; \mathrm{p}<0,001)$.

Tabla 5. Asociación entre el total de AV por subtipos y la puntuación total del CDI

\begin{tabular}{llccccc}
\hline & & $B$ & $\beta$ & $t$ & $p$ & $I C 95 \%(B)$ \\
\hline Total AV familiar & Positivos & 0,218 & 0,032 & 1,314 & 0,189 & $-0,107-0,543$ \\
& Negativos & 0,416 & 0,123 & 4,426 & $0,001 *$ & $0,232-0,600$ \\
\multirow{2}{*}{ Total AV escolar } & Positivos & $-1,685$ & $-0,152$ & $-6,339$ & $0,001 *$ & $-2,206--1,163$ \\
& Negativos & 1,227 & 0,196 & 7,480 & $0,001 *$ & $0,905-1,549$ \\
Total AV social & Positivos & $-0,229$ & $-0,036$ & $-1,418$ & 0,157 & $-0,545-0,088$ \\
& Negativos & $-0,153$ & $-0,020$ & $-0,715$ & 0,475 & $-0,572-0,267$ \\
Total AV salud & Positivos & 1,690 & 0,034 & 1,423 & 0,155 & $-0,640-4,020$ \\
& Negativos & 0,985 & 0,069 & 2,535 & $0,011 *$ & $0,223-1,747$ \\
\hline
\end{tabular}

Nota. Regresión múltiple ajustada por sexo, edad, puntuación ansiosa (SCARED) y procedencia (España u otros). IC = intervalo de confianza.

\section{Niveles de afectación según subtipos de AV y sintomatología depresiva}

La Tabla 6 incluye el modelo final que valora el grado de afectación por subtipos de AV y la puntuación total del CDI. Se obtuvieron interacciones significativas entre la edad del sujeto y el nivel de afectación/impacto negativo por AV social $(p<0,001)$ y nivel de afectación/ impacto negativo por AV salud $(p<0,05)$. Por consiguiente, se han estimado los efectos simples de ambas variables independientes para los distintos grupos de edad del estudio. Los resultados obtenidos indican que las relaciones son significativas para todas las edades, excepto los 14 años. En concreto, se observa que a los 13, 15 y 16 años el nivel de afectación negativa por AV social disminuye la puntuación en sintomatología depresiva, mientras que el impacto por AV de salud negativo aumenta la puntuación de depresión. Los niveles de afectaciones por AV familiares y escolares, tanto positivos como negativos, no se relacionaron significativamen- te con la sintomatología depresiva, así como tampoco los niveles de afectación por AV sociales y de salud positivos. El modelo que se representa en la Tabla 6 tiene una capacidad predictiva global muy buena, con $R^{2}{ }_{\text {ajustada }}=$ $0,343(F=2,069 ; p<0,024)$.

\section{DISCUSIÓN}

Investigaciones anteriores han documentado la relación existente entre los AV y la sintomatología depresiva (Williamson et al., 1998; Olsson et al., 1999; Goodyer et al., 2001). El primer propósito del presente estudio ha sido determinar dicha relación en población adolescente española, haciendo distinción entre los AV vivenciados como positivos, los AV negativos, $\mathrm{y}$ el nivel de impacto percibido de cada uno de los AV. Los resultados obtenidos nos indican que existe relación entre el número total de AV vividos y la sintomatología depresiva. Concretamente, se observa, como en otras investigaciones 
Tabla 6. Asociación entre el nivel de afectación de AV por subtipos y la puntuación total en el CDI.

\begin{tabular}{|c|c|c|c|c|c|c|c|}
\hline \multirow{2}{*}{\multicolumn{2}{|c|}{ Afectación positiva AV familiar }} & \multicolumn{2}{|l|}{$B$} & \multirow{2}{*}{$\frac{t}{-1,133}$} & \multirow{2}{*}{$\frac{p}{0,257}$} & \multicolumn{2}{|c|}{$I C 95 \%(B)$} \\
\hline & & $-0,071$ & $-0,031$ & & & $-0,194$ & 0,052 \\
\hline \multicolumn{2}{|c|}{ Afectación negativa AV familiar } & 0,055 & 0,046 & 1,695 & 0,090 & $-0,009$ & 0,119 \\
\hline \multicolumn{2}{|c|}{ Afectación positiva AV escolar } & 0,057 & 0,017 & 0,649 & 0,517 & $-0,116$ & 0,230 \\
\hline \multicolumn{2}{|c|}{ Afectación negativa AV escolar } & 0,011 & 0,006 & 0,207 & 0,836 & $-0,095$ & 0,117 \\
\hline \multicolumn{2}{|c|}{ Afectación positiva AV social } & $-0,056$ & $-0,030$ & -0.920 & 0,358 & $-0,176$ & 0,064 \\
\hline \multicolumn{2}{|c|}{ Afectación positiva AV salud } & $-0,110$ & $-0,033$ & $-0,993$ & 0,321 & $-0,328$ & 0,108 \\
\hline \multirow[t]{2}{*}{ Edad: 13años } & Afectación negativa AV sociales & $-0,164$ & $-0,320$ & $-2,405$ & $0,012 *$ & $-0,292$ & $-0,036$ \\
\hline & Afectación negativa AV salud & 0,175 & 0,249 & 1,001 & $0,036^{*}$ & 0,011 & 0,338 \\
\hline \multirow[t]{2}{*}{ Edad: 14 años } & Afectación negativa AV sociales & $-0,020$ & $-0,010$ & $-0,307$ & 0,759 & $-0,146$ & 0,106 \\
\hline & Afectación negativa AV salud & 0,091 & 0,039 & 1,184 & 0,237 & $-0,060$ & 0,241 \\
\hline \multirow[t]{2}{*}{ Edad: 15 años } & Afectación negativa AV sociales & $-0,272$ & $-0,136$ & $-2,509$ & $0,012^{*}$ & $-0,485$ & $-0,059$ \\
\hline & Afectación negativa AV salud & 0,295 & 0,128 & 2,331 & $0,020 *$ & 0,047 & 0,544 \\
\hline \multirow[t]{2}{*}{ Edad: 16 años } & Afectación negativa AV sociales & $-0,525$ & $-0,262$ & $-2,918$ & $0,004 *$ & $-0,878$ & $-0,172$ \\
\hline & Afectación negativa AV salud & 0,500 & 0,216 & 2,397 & $0,017^{*}$ & 0,091 & 0,910 \\
\hline
\end{tabular}

Nota. Regresión múltiple ajustada por sexo, edad, puntuación ansiosa (SCARED) y procedencia (España u otros). IC $=$ intervalo de confianza.

(Williamson et al., 1998; Olsson et al., 1999; Goodyer, 2001) que los AV negativos incrementan la presencia de sintomatología depresiva; sin embargo, en este caso y debido al tipo de población en el que se ha realizado la investigación, se percibe que el lugar de procedencia es una variable moderadora del efecto y que por tanto la relación es diferente si la persona es autóctona, o si por el contrario es inmigrante no nacido en España. La variabilidad en esta relación reside en que es mayor el incremento que generan los AV en la presencia de sintomatología depresiva en la población española que en la población inmigrante. Esto, en contra de lo que se esperaba, que era un incremento mayor en sintomatología depresiva en la población inmigrante debido al mayor número de $\mathrm{AV}$ que éstos han vivido, podría explicarse a partir de la teoría, anteriormente mencionada, de la resiliencia. ¿Será que los autóctonos que han debido hacer frente en su vida a menos AV no han desarrollado las estrategias de afrontamiento suficientes? ¿o será que algunos inmigrantes poseen un factor genético que les facilita una mayor resiliencia? El estudio revolucionario de la interacción gen-entorno publicado por Caspi et al. (2002) señala que la combinación de ambos factores sería determinante en la relación entre la vulnerabilidad y la resiliencia. El genotipo podría modular la sensibilidad al entorno y éste sería la respuesta al puzzle que ofrecería una respuesta al porqué algunas víctimas de maltrato no sufren efectos ulteriores y otras sí (McDermott, 2011).

Por otra parte, la relación entre el número total de AV positivos y la sintomatología depresiva no se encuentra moderada ni por la edad, ni por el lugar de procedencia y, en contra de lo que señalan Moreno et al. (1996), se observa que el número total de $\mathrm{AV}$ positivos reduce la sintomatología depresiva, es decir, que a más AV positivos vividos menor es la presencia de sintomatología depresiva.

En cuanto al impacto percibido por los $\mathrm{AV}$, los resultados indican que no existe relación significativa entre éstos y la sintomatología depresiva, ya sean los AV negativos o positivos. Algunos autores, que centran sus trabajos en la relación entre los AV y la sintomatología ansiosa (Goodyer, 1990; Moreno, Del Barrio y Mestre, 1995) explican que es más relevante en la relación con la sintomatología ansiosa el haber vivido o no un acontecimiento estresante que el impacto percibido por él, es decir, se relaciona más con la sintomatología ansiosa el haber vivido muchas situaciones estresantes aunque el 
impacto percibido sea bajo, que haber vivido sólo una situación estresante con una afectación elevada. Esta explicación, a la vista de los resultados podría extrapolarse a la sintomatología depresiva.

Otro objetivo de esta investigación ha sido valorar la relación entre los subtipos de AV (familiar, escolar, social y de salud) y la sintomatología depresiva. Los resultados muestran que para el número total de $\mathrm{AV}$ sociales, tanto positivos como negativos, no hay relación significativa con la sintomatología depresiva, cuando investigaciones como la de Jiménez et al. (2008) indicaron que la sintomatología depresiva estaba ampliamente relacionada con los $\mathrm{AV}$, específicamente con los relacionados con los iguales. Una posible explicación a esta discrepancia es que en esta investigación los AV sociales se refieren a una compilación de acontecimientos de pareja, jurídicos, y de ocio, y no sólo a aspectos centrados en las relaciones entre iguales. Además, en algunas investigaciones como la de Olsson et al. (1999) y Puskar et al. (1999) se incluye el subtipo escolar dentro del social, mientras que en este caso se trata de dos subgrupos independientes. En el caso de los AV familiares, tan sólo los negativos presentan una relación significativa con la sintomatología depresiva, aumentando su presencia, aspecto que coincide con estudios previos (Olsson et al., 1999; Puskar et al., 1999; Jiménez et al., 2008). Lo mismo ocurre con los AV de salud, en los que los positivos no presentan una relación significativa, mientras que los negativos si, aumentando estos últimos la presencia de sintomatología depresiva. Finalmente, en cuanto al número total de $\mathrm{AV}$ escolares, se puede afirmar que tanto los positivos como los negativos están relacionados con la sintomatología depresiva, disminuyendo los primeros y aumentando los segundos la presencia de dichos síntomas. Por tanto, haber tenido AV escolares positivos podría ser un factor protector pues disminuye la sintomatología depresiva, mientras que haber tenido AV negativos podría ser un factor de riesgo, pues la aumenta. Cabe señalar que probablemente los items que hacen referencia a AV escolares negativos, como es el caso del bullying, son demasiado escasos en el LEC, aunque cabe destacar que en publica- ciones recientes se ha relacionado el genotipo también con el desarrollo de problemas emocionales en las víctimas de bullying (Sugden et al., 2010), por lo que no serían los AV los que aisladamente fueran responsables de la sintomatología depresiva.

Dentro de este segundo objetivo se valoró también si el nivel de impacto percibido en cada subtipo de AV se relacionaba con sintomatología depresiva. Los resultados señalan que para los subgrupos familiar y escolar el nivel de afectación por AV positivos y negativos no tiene relación significativa con la sintomatología depresiva, lo que coincide con los resultados obtenidos en relación con el primer objetivo, y por tanto puede explicarse por la misma razón. En cuanto a los niveles de afectación por AV negativos de salud y sociales, se observa que la edad es una variable moderadora del efecto y que por tanto las relaciones entre ellos y la sintomatología depresiva varían en función de la edad que tengan los participantes. Se observa que los AV sociales negativos durante la adolescencia disminuyen la presencia de sintomatología depresiva (a excepción del grupo de adolescentes de 14 años, en el que no se observa). Esta incoherencia en relación a los resultados obtenidos en el resto de los subtipos de AV y en relación a otras investigaciones (Moreno et al., 1996; Jiménez et al., 2008; Mayer et al., 2009) podría explicarse por la hipótesis de que a esta edad los acontecimientos sociales son tan importantes, que ya sean estos positivos o negativos, el hecho de tenerlos implica la existencia de relaciones sociales, y por tanto de pertenencia a un grupo, y esto podría actuar como un factor protector ante la presencia de la sintomatología depresiva, tal y como lo son los AV positivos por sí solos.

Finalmente, en el caso del impacto percibido por los AV de salud negativos, éstos se relacionan significativamente con un aumento de la presencia de sintomatología depresiva, lo que coincide con lo expuesto por Olsson et al. (1999), los cuales establecen que entre los cinco acontecimientos más relacionados con la depresión en adolescentes se encuentran los AV relativos a la salud.

Una de las características a destacar del proyecto es el alto porcentaje de participación 
$(80,1 \%)$, muy superior a los valores referidos usualmente en estudios epidemiológicos con población comunitaria, que suelen oscilar en torno al 50\% (Ayuso et al., 2001). Otra característica es la singularidad de la muestra, pues el hecho de que el $21,2 \%$ de la población sea inmigrante ha permitido obtener datos de este grupo poblacional que durante la última década ha aumentado considerablemente en territorio español, y del que a pesar de ello tenemos escasos datos.

Los resultados de este estudio poseen implicaciones prácticas en el ámbito de la evaluación psicológica de los sujetos, así como también sobre el desarrollo de programas de prevención e intervención psicosocial. En primer lugar, la vivencia de sucesos vitales no siempre debe ser interpretada como una medida de adversidad generadora de sintomatología depresiva, pues los valores depresivos autoinformados por los sujetos parecen depender de la autopercepción de dichos eventos como positivos o negativos y de su nivel de afectación/impacto. En segundo lugar, y en relación a lo anterior, se hace necesario que los programas de evaluación y prevención valoren con precisión los aspectos estructurales de los acontecimientos vitales y su interacción con otros aspectos bio-psicosociales tales como la procedencia de los sujetos y su edad.

Una limitación del estudio es que se midieron los AV acontecidos únicamente en los últimos 12 meses, y es posible que sucesos o situaciones que hayan ocurrido en un espacio temporal más amplio sigan afectando al individuo en la actualidad y no tenemos constancia de ellos. La segunda limitación tiene que ver con la generabilidad de los resultados: la población de Rubí es una población particular puesto que los sujetos que forman parte del grupo de procedencia autóctona son en su mayoría hijos de una población que inmigró a Cataluña en las décadas de los 40, 50 y 60 desde otros puntos del país (concretamente de tierras del sur como Andalucía y Murcia), y esto les diferencia de otras poblaciones de su alrededor.

\section{REFERENCIAS}

Avenevoli, S., Stolar, M., Li J., Dierker L., \& Merikangas, K. (2001). Comorbidity of depression in children and adolescents: models and evidence from a prospective high-risc family study. Biological Psychiatry, 49, 10711081.

Ayuso, J., Vazquez, J., Dowrick, C., Lehtinen, V., Dalgard, O., Casey, P., Wilkinson, C., Lasa, L., Page, H., Dunn, G., \& Wilkinson, G. (2001). Depressive disorders in Europe: prevalence figures from the ODIN study. British Journal of Psychiatry, 179, 308-316.

Birmaher, B., Khetarpal S., \& Cully M. (1997). The Screen for child Anxiety Related Emotional Disorders (SCARED): Scale construction and psychometric characteristics. Journal of the American Academy of Child \& Adolescent Psychiatry, 36, 545-553.

Brand, A. H., \& Jonhson, J. H. (1982). Note on reliability of the Life Events Checklist. Psychological Reports, 50, 1274.

Del Barrio, M., Moreno-Rosset, C., y López-Martínez, R. (1999). El Children's Depression Inventory (CDI: Kovacs, 1992). Su aplicación en población española. Clínica y Salud, 10, 393-416.

Caspi, A., McClay, J., Moffitt, T., Mill, J., Martin, J., Craig, I., \& Poulton, R. (2002). Role of genotype in the cycle of violence in maltreated children. Science (New York), 297, 851-854.

Coddington, R. (1972). The significance of life events as etiologic factors in the diseases of children: I. A survey of professional workers. Journal of Psychosomatic Research, 16, 7-18.

Doval, E., Martínez, M. y Domènech, E. (2011). Evidencias de calidad psicométrica de la versión en castellano del screen for child anxiety related emotional disorders (SCARED) de 41 items. Ansiedad y Estrés, 17, 199-210.

Domènech, E., Subirà, S., y Cuxart, F. (1996). Trastornos del estado de ánimo en la adolescencia temprana: La labilidad afectiva. En J. Buendía (Ed.), Psicopatología en niños y adolescentes: Desarrollos actuales. Madrid: Pirámide.

Egeland, B., Carlson, E., \& Sroufe, L. (1993). Resilience as process. Special issue: Milestones in the development of resilience. Development and Psychopathology, 5, 517-528.

Finlay-Jones, R., \& Brown, G. (1981). Types of stressful life events and the onset of anxiety and depressive disorders. Psychological Medicine, 11, 803-815.

Ge, X., Coger, R., \& Elder, G. (2001). Pubertal transition, stressful life events, and the emergence of gender differences in adolescent depressive symptoms. Developmental Psychology, 37, 404-417.

Goodyer, I. (1990). Recent life events and psychiatric disorder in school age children. Journal of Child Psychology and Psychiatry, 31, 839-848. 
Goodyer, I. (2001). Life events: Their nature and effects. In I. M. Goodyer (Ed.), The depressed child and adolescent ( $2^{\text {nd }}$ ed., pp. 204-232). Cambridge: Cambridge UniversityPress.

Goodyer, I., Herbert, J., \& Tamplin, A. (2000). First episode major depression in adolescents: Affective, cognitive and endocrine characteristics of risk status and predictors of onset. British Journal of Psychiatry, 176, 142-149.

Goodyer, I., Kolvin, I., \& Gatzanis, S. (1985). Recent undesiderable life events and psychiatric disorder in childhood and adolescence. Journal of Child Psychology and Psychiatry, 147, 517-523.

Hjemdal, O., Aune, T., Reinfjell, T., Stiles, T., \& Friborg, O. (2007). Resilience as a predictor of depressive symptoms: a correlational study with young adolescents. Clinical Child Psychology and Psychiatry, 12, 91-104.

Hollingshead, A. (1975). Four factor index of social status. Manuscrito no publicado. Departamento de Sociología, Universidad de Yale.

Instituto Nacional de Estadística (2009). Recuperado el 20 de noviembre de 2009, de http://www.ine.es/.

Jiménez, L., Menéndez, S., e Hidalgo, M. (2008). Un análisis de los acontecimientos vitales estresantes durante la adolescencia. Apuntes de Psicología, 26, 427-440.

Johnson, J. (1986). Life events as stressors in childhood and adolescence. Beverly Hills: SAGE.

Johnson, J., \& McCutcheon, S. (1980). Assessing life stress in older children and adolescents: Preliminary finding with the Life Events Checklist. In I. G. Sarason and C.D. Spielberger (Eds.), Stress and Anxiety (pp. 111-125). Washington, DC: Hemisphere.

Kovacs, M. (1983). The Children's Depression Inventory: A self-rated depression scale for school-aged youngsters. University of Pittsburgh. Manuscrito no publicado.

Kovacs, M. (1990). Comorbid anxiety disorders in childhood-onset depressions. In J. D. Maser, C. Cloninger, J. D. Maser, C. Cloninger (Eds.), Comorbidity of mood and anxiety disorders (pp. 271-281). Washington, DC US: American Psychiatric Association.

Kovacs, M. (1992). Children's Depression Inventory (CDI). Toronto: MultiHealth Systems, Inc.

Larson, R., \& Ham, M. (1993). Stress and 'storm and stress' in early adolescence: The relationship of negative life-events with disphorie affect. Developmental Psychology, 29, 130-140.

Lázaro, S. (2009). Resiliencia en niños y adolescentes: Revisión teórica e implicaciones para la intervención psicoeducativa en situaciones de maltrato familiar. Estudios de Psicología, 30, 89-104.

Luthar, S., Cicchetti, D., \& Becker, B. (2000) The construct of resilience: A critical evaluation and guidelines for future work. Child Development, 71, 543-562.
McDermott, J. F. (2011). The Longitudinal Study: A Bridge to the Future. Journal of the American Academy of Child \& Adolescent Psychiatry, 50, 317-319.

Masten A., \& Reed, M. (2002). Resilience in development. In C. R. Snyder and S. J. López (Eds.), Handbook of positive psychology (pp. 74-88). New York: Oxford University Press.

Mayer, L., Lopez-Duran, N., Kovacs, M., George, C., Baji, I., Kapornai, K., Kiss, E., \& Vetró, A. (2009). Stressful life events in a clinical sample of depressed children in Hungary. Journal of Affective Disorders, 115, 207-214.

Mestre, V., Frías, D., y García-Ros, R. (1992). Propiedades psicométricas del Children's Depression Inventory (CDI) en población adolescente: Fiabilidad y validez. Psicológica, 13, 149-159.

Moreno, C., y Del Barrio, V. (1992). Inventario de Acontecimientos Vitales en Infancia y Adolescencia (AVIA). Manuscrito no publicado. Madrid: UNED.

Moreno, C., Del Barrio, V., y Mestre, V. (1996). Acontecimientos vitales y depresión en adolescentes. Revista electrónica de la Federación española de Asociaciones de Psicología, 1.

Moreno, C., Del Barrio, V., y Mestre, V. (1995). Ansiedad y acontecimientos vitales en adolescentes. Revista Latinoamericana de Psicología, 27, 471-496.

Muris, P., Merckelbach, H., Mayer, B., van Brakel, A., Thissen, S., Moulaert, V., \& Gadet, B. (1998). The Screen for Child Anxiety Related Emotional Disorders (SCARED) and traditional childhood anxiety measures. Journal Of Behavior Therapy And Experimental Psychiatry, 29, 327-339.

Oliva, A., Jiménez, J., Parra, A., y Sánchez-Queija, I. (2008). Acontecimientos vitales estresantes, resiliencia y ajuste adolescente. Revista de Psicopatología y Psicología Clínica, 13, 53-62.

Olsson, I., Nordström, M., Arinell, H., \& Von Knorring, A. (1999). Adolescent depression and stressful life events. Nordic Journal of Psychiatry, 53, 339-346.

Puskar, K., Tusaie-Mumford, K., Sereika, S., \& Lamb, J. (1999). Screening and predicting adolescent depressive symptoms in rural settings. Archives of Psychiatric Nursing, 13, 3-11.

Rutter, M. (2007). Resilience, competence and coping. Child Abuse and Neglect, 31, 205-209.

Sandín, B., Valiente, R.M., y Chorot, P. (2009). RCADS: Evaluación de los síntomas de los trastornos de ansiedad y depresión en niños y adolescentes. Revista de Psicopatología y Psicología Clínica, 14, 193-206.

Smith, P. (2009). Resilience: resistance factor for depressive symptom. Journal of Psychiatric And Mental Health Nursing, 16, 829-837.

Sugden, K., Arseneault, L., Harrington, H., Moffitt, T., Williams, B., \& Caspi, A. (2010). Serotonin transporter gene moderates the development of emotional problems among children following bullying victimiza- 
tion. Journal Of The American Academy Of Child And Adolescent Psychiatry, 49, 830-840.

Tiet, Q., Bird, H., Hoven, C., Moore, R., Wu, P., Wicks, J., Jensen, P., Goodman, S., \& Cohen, P. (2001). Relationship between specific adverse life events and psychiatric disorders. Journal Of Abnormal Child Psychology, 29, 153-164.

Weller, E. \& Weller, R. (2000). Depression in adolescents, growing pains or true morbidity? Journal of Affective Disorders, 61, 9-13.
Williamson, D., Birmaher, B., Frank, E., Anderson, B., Matty, M., \& Kupfer, D. (1998). Nature of life events and difficulties in depressed adolescents. Journal of the American Academy of Child and Adolescent Psychiatry, 37, 1047-1057.

Williamson, D., Birmaher, B., Dahl., R., \& Ryan, N. (2005). Stressful life events in anxious and depressed children. Journal Of Child and Adolescent Psychopharmacology, 15, 571-580. 